\title{
Correcting Systematic Energy Deficits in the Laser-pulsed Atom Probe Mass Spectrum of $\mathrm{SiO}_{2}$
}

\author{
Benjamin Caplins ${ }^{1}$, Paul Blanchard ${ }^{1}$, Ann Chiaramonti $^{1}$, David Diercks ${ }^{2}$, Luis Miaja-Avila ${ }^{1}$ and Norman \\ Sanford ${ }^{1}$
}

${ }^{1}$ NIST, Boulder, Colorado, United States, ${ }^{2}$ Colorado School of Mines, Golden, Colorado, United States

Voltage-pulsed atom probe tomography is difficult or impossible to use for materials with poor electrical conductivity. Laser-pulsed atom probe tomography overcomes this limitation by triggering field evaporation optically enabling the analysis of strongly insulating materials[1]. However, using a laserpulse to trigger ion evaporation does not completely render the electrical insulating properties of the tip irrelevant. For example, when the electrical resistivity of the tip is high enough, the atto-amp to femtoamp ion current emitted from the tip apex can result in a significant voltage drop along the tip axis (Figure 1a)[2]. This voltage drop has the effect of giving the emitted ions an "energy deficit" - i.e. the actual accelerating voltage for the ion is smaller than the voltage applied experimentally to the base of the sample. A static energy deficit is resolved when a mass spectrum is calibrated, however, when the voltage drop fluctuates throughout an atom probe dataset it cannot be corrected with standard analysis methods. This effect is noted in the literature for infrared, visible, and ultraviolet laser wavelengths[3]. More recently, it also appeared when using extreme ultraviolet (EUV) light[4] to trigger field ion evaporation of $\mathrm{SiO}_{2}$; Figure $1 \mathrm{~b}$ shows a time-of-flight history for an $\mathrm{SiO}_{2}$ tip held at constant voltage collected using a pulsed $29.6 \mathrm{~nm}$ light source. Over the course of the run, the pointing and intensity of the EUV light source fluctuated, resulting in a changing ion current and therefore a changing energy deficit, which significantly degrades the quality of the resulting mass spectrum (Figure 1f) and degrades subsequent data extraction and interpretation.

In order to correct the data for this unknown (fluctuating) acceleration voltage, we developed an empirical algorithm. The algorithm first splits the data into a large number of contiguous "chunks" - two representative chunks, "A" and "B", are shown in Figure 1b. Then, the chunks are optimally aligned to a reference spectrum with a multiplicative correction factor (Figure 1c) using a histogram-based dot product optimization metric (Figure 1e). Applying a log-transform to the time-of-flight data enables the use of the fast Fourier transform for the optimization step. The result is that a correction factor can be determined for every ion's flight time that minimizes the effect of the unknown (varying) voltage drop (Figure 1d). The resulting mass spectrum of the corrected data is significantly improved (narrower peaks with a higher maximum) versus the uncorrected data (Figure 1f). This correction algorithm ensures that the highest quality input data is supplied into the atom probe data analysis pipeline. In principle, this method can be extended to spatially heterogenous materials using a multi-reference approach, which would be facilitated by well-known unsupervised machine learning algorithms [5]. 

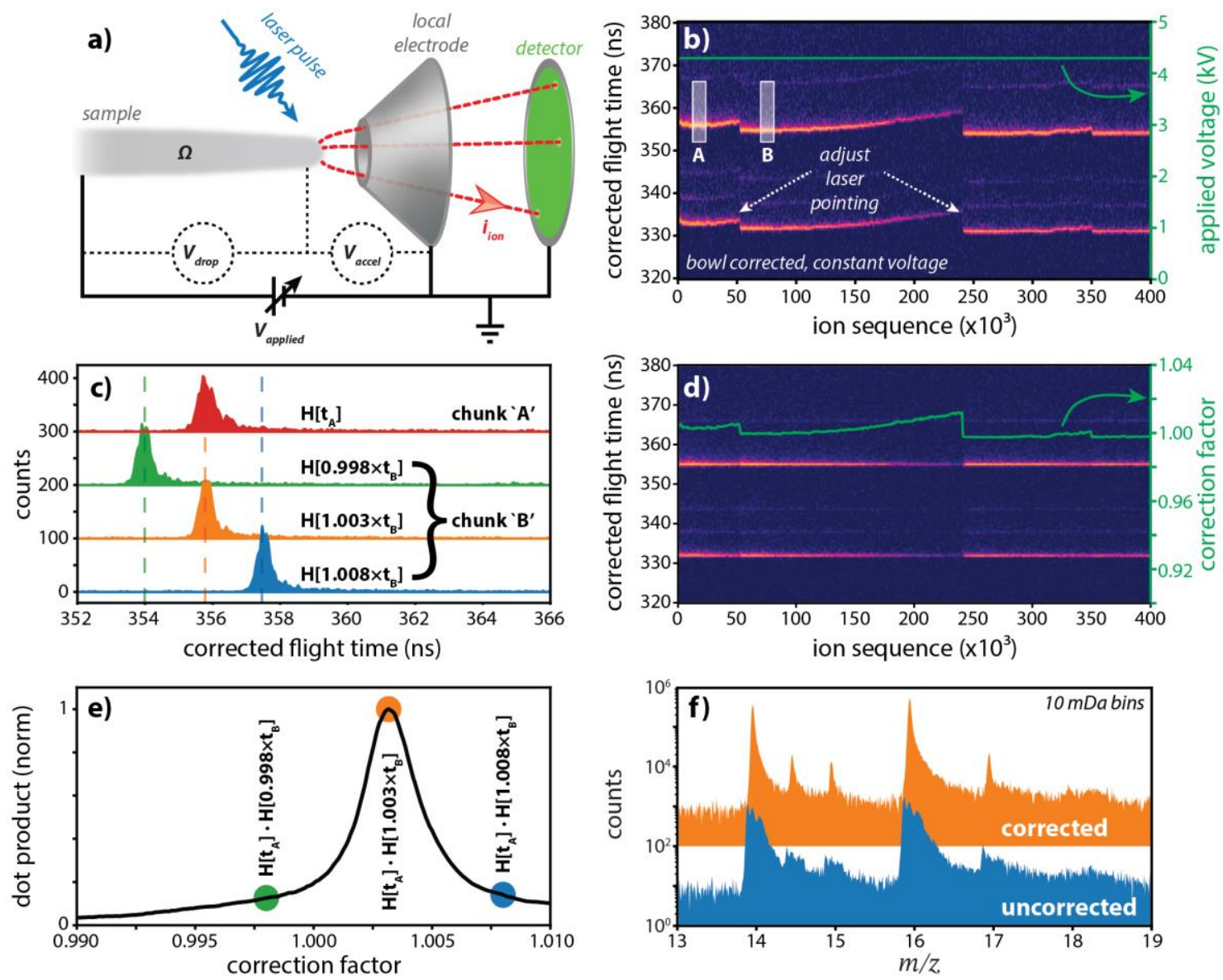

Figure 1. (a) Schematic of a laser-pulsed atom probe experimental setup. (b) Time-of-flight history of a constant voltage $\mathrm{SiO} 2$ experiment. (c-e) An empirical algorithm corrects for the unknown (time-varying) voltage drop. (f) The mass spectrum generated from the corrected data has significantly improved quality.

\section{References}

[1] B. Gault et al., Appl. Phys. Lett. 88 (2006), p. 114101.

[2] L. Arnoldi et al., J. Appl. Phys. 115 (2014), p. 203705.

[3] L. Arnoldi et al., J. Appl. Phys. 126 (2019), p 045710.

[4] A. Chiaramonti et al., MRS Adv. 4 (2019), p. 2367.

[5] This work is a contribution of the US Government and is not subject to United States copyright. 